quatrième série-tome $43 \quad$ fascicule 2 mars-avril 2010

$$
\begin{aligned}
& \text { ANNALES } \\
& \text { SCIENTIFIQUES } \\
& \text { de } \\
& \text { L'ECOLE } \\
& \text { NORMALE } \\
& \text { SUPÉRIEURE }
\end{aligned}
$$

Jérémy BLANC

Groupes de Cremona, connexité et simplicité 
Ann. Scient. Éc. Norm. Sup.

$4^{\text {e }}$ série, t. 43,2010 , p. 357 à 364

\title{
GROUPES DE CREMONA, CONNEXITÉ ET SIMPLICITÉ
}

\author{
PAR JÉRÉMY BLANC
}

RÉSumÉ. - Le groupe de Cremona est connexe en toute dimension et, muni de sa topologie, il est simple en dimension 2 .

Abstract. - The Cremona group is connected in any dimension and, endowed with its topology, it is simple in dimension 2.

\section{Questions et résultats}

Soit $k$ un corps algébriquement clos. On note $\mathrm{Cr}_{n}(k)$ le groupe de Cremona de dimension $n$, groupe des transformations birationnelles de $\mathbb{P}_{k}^{n}$, anti-isomorphe via l'action sur le corps des fractions rationnelles à $\operatorname{Aut}_{k}\left(k\left(x_{1}, \ldots, x_{n}\right)\right)$. Ce groupe est muni d'une topologie naturelle (décrite à la section 2).

En 1974, dans un rapport sur les questions ouvertes importantes en géométrie algébrique [5], D. Mumford consacre un paragraphe au groupe $\mathrm{Cr}_{2}(k)$. Il parle de mettre une topologie sur le groupe, et pose alors la question : ce groupe est-il simple? Le théorème 4.2 démontré plus bas permet de répondre par l'affirmative.

La technique utilisée pour cela est élémentaire. Elle permet également de prouver que le groupe $\mathrm{Cr}_{n}(k)$ est connexe pour tout $n$ (Théorème 5.1). Ceci répond à une question posée par J-P. Serre lors du $1000^{\mathrm{e}}$ exposé Bourbaki [8], concernant la dimension $n \geq 3$, le cas $n \leq 2$ étant déjà bien connu.

Cet article est articulé ainsi : la section 2 donne des rappels sur la topologie de Zariski de $\mathrm{Cr}_{n}(k)$, la section 3 présente un lemme de déformation, qui permet de montrer la simplicité de $\mathrm{Cr}_{2}(k)$ (section 4) et la connexité de $\mathrm{Cr}_{n}(k)$ (section 5).

Je tiens à remercier J.-P. Furter pour des discussions intéressantes sur cet article, et tout spécialement J-P. Serre pour ses relectures attentives de cet article et ses précieuses corrections. 


\section{La topologie de Zariski de $\mathrm{Cr}_{n}(k)$}

Soit $X$ une $k$-variété ( $k$ est toujours le corps algébriquement clos fixé au départ). On note $\operatorname{Bir}(X)$ l'ensemble des applications birationnelles $X \rightarrow X$, et $\operatorname{Aut}(X) \subset \operatorname{Bir}(X)$ le groupe des automorphismes de $X$.

Afin de décrire la topologie de $\operatorname{Bir}(X)$, décrivons tout d'abord les morphismes $A \rightarrow \operatorname{Bir}(X):$

Définition 2.1. - Une famille algébrique d'éléments de $\operatorname{Bir}(X)$ est la donnée d'une application rationnelle $f: A \times X \rightarrow X$ où $A$ est une $k$-variété, définie sur un ouvert dense $U$ tel que, pour tout $a \in A, U_{a}:=U \cap(\{a\} \times X)$ soit un ouvert dense de $\{a\} \times X$ et que la restriction de id $\times f$ à $U$ soit un isomorphisme de $U$ sur un ouvert dense de $A \times X$.

Pour tout $a \in A$, l'application birationnelle $x \rightarrow f(a, x)$ représente alors un élément $f_{a} \in \operatorname{Bir}(X)$. La famille $f_{a}(a \in A)$ représente une application $A \rightarrow \operatorname{Bir}(X)$, que l'on appellera morphisme de $A$ vers $\operatorname{Bir}(X)$.

Cette définition correspond à celle de [8] et [2, §1]; un morphisme $A \rightarrow \operatorname{Bir}(X)$ correspond alors à un pseudo-automorphisme du $A$-schéma $A \times X$. On définit la topologie de Zariski sur $\operatorname{Bir}(X)$ de la manière suivante (voir $[8, \S 1.6])$ :

Définition 2.2. - On dit qu'un ensemble $R \subset \operatorname{Bir}(X)$ est fermé si, pour toute $k$-variété $A$ et tout morphisme $A \rightarrow \operatorname{Bir}(X)$, la préimage de $R$ dans $A$ est fermée.

Comme l'explique [8], ceci donne une topologie sur $\operatorname{Bir}(X)$, qui est la topologie la plus fine qui rende les morphismes vers $\operatorname{Bir}(X)$ continus. De plus, en définissant de manière analogue la topologie de Zariski sur $\operatorname{Bir}(X) \times \operatorname{Bir}(X)$, la composition donne une application continue $\operatorname{Bir}(X) \times \operatorname{Bir}(X) \rightarrow \operatorname{Bir}(X)$.

En particulier, on peut restreindre ceci à $\operatorname{Aut}(X)$ et mettre ainsi une topologie sur ce groupe. Lorsque $X=\mathbb{P}_{k}^{n}$, on peut démontrer que l'on retrouve la topologie de Zariski habituelle du groupe algébrique $\operatorname{Aut}(X)=\operatorname{PGL}(n+1, k)$ et qu'en fait $\operatorname{Aut}(X) \rightarrow \mathrm{Cr}_{n}(X)$ est une immersion fermée [7].

Les groupes qui nous intéressent le plus sont ceux où la $k$-variété $X$ est rationnelle. On rappelle que si $X$ est rationnelle, de dimension $n$, alors $\operatorname{Bir}(X)$ s'identifie naturellement à $\operatorname{Cr}_{n}(k)=\operatorname{Bir}\left(\mathbb{P}_{k}^{n}\right)$ via une application birationnelle choisie $X \rightarrow \mathbb{P}_{k}^{n}$; le choix de celleci fait juste varier l'homéomorphisme $\operatorname{Bir}(X) \rightarrow \mathrm{Cr}_{n}(k)$. Dans la suite, on prendra le plus souvent $X=\mathbb{A}_{k}^{n}$ ou $X=\mathbb{P}_{k}^{n}$, suivant les besoins.

\section{Préliminaires techniques}

\subsection{Le groupe de de Jonquières}

Pour $n \geq 2$, notons $\phi$ la projection

$$
\left(x_{0}: \cdots: x_{n}\right) \rightarrow\left(x_{1}: \cdots: x_{n}\right)
$$

de $\mathbb{P}_{k}^{n}$ dans $\mathbb{P}_{k}^{n-1}$. On appelle groupe de de Jonquières $J_{n}$ le sous-groupe de $\operatorname{Bir}\left(\mathbb{P}_{k}^{n}\right)$ qui préserve l'ensemble des fibres de $\phi$. On note $J_{n}^{0}$ le sous-groupe de $J_{n}$ constitué des éléments qui préservent une fibre générale de $\phi$. 
De manière affine, on peut restreindre $\phi$ à la projection $k^{n} \rightarrow k^{n-1}$, et ainsi voir que $J_{n}$ est naturellement isomorphe à $J_{n}^{0} \rtimes \operatorname{Bir}\left(k^{n-1}\right)$, où

$$
J_{n}^{0} \simeq \operatorname{Aut}\left(\mathbb{P}_{K}^{1}\right) \simeq \operatorname{PGL}(2, K), \text { avec } K=k\left(x_{1}, \ldots, x_{n-1}\right) .
$$

L'homomorphisme déterminant $\mathrm{GL}(2, K) \rightarrow K^{*}$ induit un homomorphisme surjectif

$$
\text { det: } \operatorname{PGL}(2, K) \rightarrow K^{*} /\left(K^{*}\right)^{2},
$$

où $\left(K^{*}\right)^{2}$ désigne l'ensemble des carrés de $K^{*}$. On notera $J_{n}^{1} \subset J_{n}^{0}$ le sous-groupe normal correspondant au noyau de det. Alors, l'homomorphisme précédent nous donne

$$
J_{n}^{1} \simeq \operatorname{PSL}(2, K) .
$$

Le groupe $J_{n}^{1}$ est simple [3, Chapitre II, §2]. De plus, comme tout élément $f \in J_{n}^{0}$ satisfait $\operatorname{det}\left(f^{2}\right)=1$, le quotient $J_{n}^{0} / J_{n}^{1}$ est un groupe abélien de type $(2, \ldots, 2, \ldots)$. Les classes de $J_{n}^{0}\left(\bmod J_{n}^{1}\right)$ sont représentées par les involutions de de Jonquières $f_{h}:\left(x_{1}, \ldots, x_{n}\right) \rightarrow\left(x_{1}, \ldots, x_{n-1}, h / x_{n}\right)$, où $h \in k\left(x_{1}, \ldots, x_{n-1}\right)^{*}=K^{*}$. Puisque $\operatorname{det}\left(f_{h}\right)=-h$, deux involutions $f_{h}$ et $f_{h^{\prime}}$ représentent la même classe si et seulement si $h / h^{\prime}$ est un carré dans $K^{*}$.

\subsection{Dérivée normale}

Dans cette section, on se donne la situation suivante :

Partons d'une $k$-variété lisse $X$. Soit $Y$ la droite affine sur $k$ et soit $Z=X \times Y$; le morphisme $x \mapsto(x, 0)$ identifie $X$ à une sous-variété de $Z$. Soit $U$ un ouvert dense de $Z$ tel que $U_{X}:=U \cap X$ soit dense dans $X$ et soit $f: U \rightarrow Z$ un morphisme qui envoie $U_{X}$ dans $X$; notons $f_{X}: U \rightarrow X$ et $f_{Y}: U \rightarrow Y$ les deux composantes de $f$.

À partir de cette donnée, on va définir la dérivée normale de $f$, qui est une application rationnelle $f_{0}: Z \rightarrow Z$, et montrer qu'il s'agit d'une limite de conjugués de $f$.

La fonction $f_{Y}$ a la propriété que $f_{Y}(x, y)=0$ si $y=0$; on en déduit que $f_{Y}$ est divisible par la fonction « $y$ », ce qui veut dire que $f_{Y}(x, y)=y \cdot g_{Y}(x, y)$ pour une certaine fonction $g_{Y}$ sur $U$. Ceci nous permet de définir la dérivée normale de $f$ le long de $X$, qui est le morphisme

$$
f_{0}: U_{X} \times Y \rightarrow Z
$$

donné par la formule $f_{0}(x, y)=\left(f_{X}(x, 0), y \cdot g_{Y}(x, 0)\right)$.

On remarque que $f_{0}$ ne dépend que du comportement de $f$ dans un voisinage infinitésimal de $X$ et est une sorte de linéarisation de $f$; en fait $f_{0}$ ne dépend pas du choix de l'ouvert $U$ mais seulement de $f$ vue comme application rationnelle de $Z$ dans lui-même. De plus $(x, 0) \mapsto f_{X}(x, 0)$ est la restriction de $f$ à $X$, ce qui implique que $f_{0}$ est compatible avec la projection $Z \rightarrow X$; on a les diagrammes commutatifs

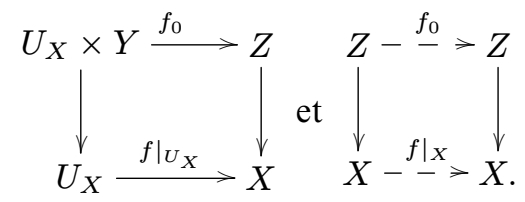

De plus, $f_{0}$ est une homothétie sur chaque fibre.

Montrons maintenant que $f_{0}$ est une limite de conjugués de $f$. Soit $T$ un autre exemplaire de la droite affine sur $k$; pour tout $t \in T$, soit $U_{t}$ l'ouvert de $Z$ formé des $(x, y)$ tels que $(x, t y)$ 
appartienne à $U$. Remarquons que $U_{0}=U_{X} \times Y$. La réunion $U_{T}$ des $\{t\} \times U_{t}$ est un ouvert de $T \times Z$ contenant $T \times U_{X}$. Si $t \neq 0$, soit $s_{t}$ l'automorphisme $(x, y) \mapsto(x, t y)$ de $Z$ et posons $f_{t}=s_{t}^{-1} \circ f \circ s_{t}$, ce qui a un sens sur $U_{t}$; si $t=0$, définissons $f_{t}=f_{0}$ comme ci-dessus; c'est un morphisme défini sur $U_{0}=U_{X} \times Y$.

Lemme 3.1. - Avec les notations précédentes, la famille des $f_{t}(t \in T)$ définit un morphisme $F: U_{T} \rightarrow Z$.

Démonstration. - On a $F(t, x, y)=\left(f_{X}(x, t y), y \cdot g_{Y}(x, t y)\right)$ : lorsque $t \neq 0$, cela résulte de $t^{-1} f_{Y}(x, t y)=y \cdot g_{Y}(x, t y)$ et lorsque $t=0$, c'est la définition de $f_{0}$. Le lemme suit alors $\mathrm{du}$ fait que $(t, x, y) \mapsto f_{X}(x, t y)$ et $(t, x, y) \mapsto g_{Y}(x, t y)$ sont des morphismes définis sur $U_{T}$.

Lemme 3.2. - Avec les mêmes notations qu'avant, supposons de plus que X est irréductible, que $f$ est un isomorphisme de $U$ sur un ouvert $V$ de $Z$ et que $f$ se restreint à un isomorphisme de $U_{X}=U \cap X$ vers $V_{X}=V \cap X$ (ce qui implique que $f \in \operatorname{Bir}(Z)$ et $\left.f\right|_{X} \in \operatorname{Bir}(X)$ ).

Alors, la famille $f_{t}(t \in T)$ définit un morphisme $T \rightarrow \operatorname{Bir}(Z)$ (au sens de la définition 2.1).

Démonstration. - Le lemme 3.1 montre que $F: U_{T} \rightarrow Z$ est un morphisme, qui induit donc une application rationnelle $T \times Z \rightarrow Z$. Pour tout $t \in T, U_{T} \cap(\{t\} \times Z)$ n'est rien d'autre que $\{t\} \times U_{t}$, ouvert dense de $\{t\} \times Z$ par construction, et la restriction de $F$ à cet ouvert correspond à $f_{t}$.

Notons $r: V \rightarrow U$ l'inverse de $f$, qui applique $V_{X}=V \cap X$ dans $X$ par construction, et utilisons la construction précédente pour $r=\left(r_{X}, r_{Y}\right)$. On a $V_{t}=\{(x, y) \in Z \mid(x, t y) \in V\}$ et le lemme 3.1 nous donne un morphisme $R: V_{T} \rightarrow Z$, dont la restriction à $\{t\} \times V_{t}$ correspond à $r_{t}$.

Il suffit alors de voir que id $\times f$ est un isomorphisme de $U_{T}$ vers $V_{T}$, dont l'inverse est id $\times r$, ce qui peut par exemple se déduire de la forme explicite de $F(t, x, y)$ et $R(t, x, y)$ donnée dans la preuve du lemme 3.1.

\subsection{Le lemme de déformation appliqué au groupe de Cremona}

Rappelons que si $Z$ est une $k$-variété irréductible lisse, si $f \in \operatorname{Bir}(Z)$ et $H, H^{\prime} \subset Z$ sont deux hypersurfaces irréductibles, on dit que $f$ se restreint $\grave{a}$ une application birationnelle $\left.f\right|_{H}: H \rightarrow H^{\prime}$ si $f$ est définie sur un ouvert $U$ tel que $U \cap H$ soit un ouvert dense de $H$ et tel que $\left.f\right|_{U \cap H}: U \cap H \rightarrow H^{\prime}$ soit une immersion ouverte. On peut également présenter cette notion de la façon suivante : les hypersurfaces $H$ et $H^{\prime}$ définissent des valuations discrètes $v$ et $v^{\prime}$ du corps des fonctions de $Z$, et l'on demande que $f$ transforme $v$ en $v^{\prime}$.

En appliquant les résultats de la section 3.2 au cas d'une transformation birationnelle de $\mathbb{P}_{k}^{n}$, nous allons démontrer le résultat suivant.

Lemme 3.3. - Pour $n \geq 2$, notons $H_{0} \subset \mathbb{P}_{k}^{n}$ l'hyperplan d'équation $x_{0}=0$. Soit $f \in \operatorname{Bir}\left(\mathbb{P}_{k}^{n}\right)$ un élément qui se restreint à une application birationnelle $\left.f\right|_{H_{0}}: H_{0} \rightarrow H_{0}$.

Notons $Z \subset \mathbb{P}_{k}^{n}$ le complémentaire de l'hyperplan d'équation $x_{n}=0$ et $X=Z \cap H_{0}$, de telle sorte que $Z=X \times Y$ avec $Y \cong \mathbb{A}_{k}^{1}$.

$4^{\mathrm{e}}$ SÉRIE - TOME $43-2010-\mathrm{N}^{\mathrm{o}} 2$ 
Alors, en reprenant les notations de la section 3.2 , la dérivée normale $f_{0}$ de $f$ le long de $X$ est un élément de $J_{n}$ tel que le diagramme suivant soit commutatif

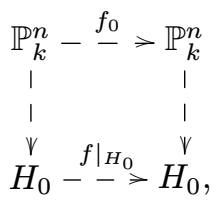

où les flèches verticales correspondent à la projection $\left(x_{0}: x_{1}: \cdots: x_{n}\right) \rightarrow\left(0: x_{1}: \cdots: x_{n}\right)$. Le morphisme donné par le lemme 3.2

$$
\begin{aligned}
\mathbb{A}_{k}^{1} \cong T & \rightarrow \operatorname{Bir}(Z) \cong \operatorname{Bir}\left(\mathbb{P}_{k}^{n}\right) \\
t & \mapsto f_{t}
\end{aligned}
$$

envoie 0 sur $f_{0}, 1$ sur $f_{1}=f$ et $t \neq 0$ sur $f_{t}=s_{t}^{-1} \circ f \circ s_{t}$ où $s_{t}$ correspond ici à l'automorphisme $\left(x_{0}: x_{1}: \cdots: x_{n}\right) \mapsto\left(t x_{0}: x_{1}: \cdots: x_{n}\right)$ de $\mathbb{P}_{k}^{n}$.

Démonstration. - On a $X \cong \mathbb{A}_{k}^{n-1}$ et $Z=X \times Y \cong \mathbb{A}_{k}^{n}$ est un ouvert dense de $\mathbb{P}_{k}^{n}$. On se donne deux ouverts $U, V \subset Z \subset \mathbb{P}^{n}$ tels que $f$ se restreigne à un isomorphisme $U \rightarrow V$ et également à un isomorphisme $U_{X}=U \cap X \rightarrow V_{X}=V \cap X$, où $U_{X}$ et $V_{X}$ sont denses dans $X$. On peut alors utiliser tous les résultats de la section 3.2. La projection $Z \rightarrow X$ correspond à $\phi: \mathbb{P}_{k}^{n} \rightarrow H_{0}$ donné $\operatorname{par}\left(x_{0}: x_{1}: \cdots: x_{n}\right) \rightarrow\left(0: x_{1}: \cdots: x_{n}\right)$. La commutativité du diagramme (1) entraîne donc celle de (2) et implique que $f_{0} \in J_{n}$. Le morphisme $t \mapsto f_{t}$ est donné par le lemme 3.2 et sa description ici résulte de celle donnée précédemment.

\section{Simplicité de $\operatorname{Bir}\left(\mathbb{P}_{k}^{2}\right)$}

Proposition 4.1. - Supposons $n \geq 2$. Soit $N \subset \mathrm{Cr}_{n}(k)$ un sous-groupe non trivial qui soit à la fois normal et fermé. Alors, $N$ contient $\operatorname{Aut}\left(\mathbb{P}_{k}^{n}\right) \simeq \operatorname{PGL}(n+1, k)$ et $J_{n}^{1} \simeq \operatorname{PSL}\left(2, k\left(x_{1}, \ldots, x_{n-1}\right)\right)$.

Démonstration. - Soit $h$ un élément non trivial de $N$, qui se restreint à un isomorphisme $\left.h\right|_{U}: U \rightarrow V$, où $U, V$ sont des ouverts denses de $\mathbb{P}_{k}^{n}$. Soit $p$ un point de $U$ et notons $q=h(p) \in V$ son image; on peut supposer que $q$ et $p$ sont différents. Il existe un élément $\alpha \in \operatorname{Aut}\left(\mathbb{P}_{k}^{n}\right)$ qui fixe à la fois $q$ et $p$. Par conséquent, $g=\left(\alpha h^{-1} \alpha^{-1}\right) h \in N$ fixe $p$ (et $q$ ).

Notons $T_{p}$ l'espace tangent à $p$ et $\mathbb{P}\left(T_{p}\right) \simeq \mathbb{P}_{k}^{n-1}$ son projectivisé. Alors, $g$ induit un automorphisme $g_{p} \in \operatorname{Aut}\left(\mathbb{P}\left(T_{p}\right)\right)$. Montrons maintenant que, pour un choix convenable de $\alpha$, l'automorphisme $g_{p}$ est non trivial. Comme $h$ envoie $p$ sur $q$ via un isomorphisme local, il induit un isomorphisme (linéaire) $l: \mathbb{P}\left(T_{p}\right) \rightarrow \mathbb{P}\left(T_{q}\right)$. En notant $\alpha_{p} \in \operatorname{Aut}\left(\mathbb{P}\left(T_{p}\right)\right)$ et $\alpha_{q} \in \operatorname{Aut}\left(\mathbb{P}\left(T_{q}\right)\right)$ les automorphismes induits par les actions respectives de $\alpha \operatorname{sur} \mathbb{P}\left(T_{p}\right)$ et $\mathbb{P}\left(T_{q}\right)$, on a $g_{p}=\alpha_{p} l^{-1}\left(\alpha_{q}\right)^{-1} l$. Pour que $g_{p}$ soit non trivial, il suffit par exemple de choisir $\alpha=\left(x_{0}: x_{1}: \cdots: x_{n}\right) \mapsto\left(\lambda x_{0}: x_{1}: \cdots: x_{n}\right)$, avec $\lambda \in k \backslash\{0,1\}$, si $p=(1: 0: \cdots: 0)$ et $q=(0: 1: 0: \cdots: 0)$.

Soit $\sigma:\left(x_{0}: \cdots: x_{n}\right) \rightarrow\left(\frac{1}{x_{0}}: \cdots: \frac{1}{x_{n}}\right)$ la transformation standard de $\mathbb{P}_{k}^{n}(\mathrm{de}$ degré $n$ ), alors $\sigma$ est une involution qui contracte l'hyperplan $H_{0}$ d'équation $x_{0}=0$ sur le point $(1: 0: \cdots: 0)$, et qui envoie la valuation associée à $H_{0}$ sur celle associée au diviseur exceptionnel obtenu en éclatant $(1: 0: \cdots: 0)$. En choisissant $p=(1: 0$ : 
․ 0 ) (quitte à conjuguer $g$ par un automorphisme de $\mathbb{P}_{k}^{n}$ ), $f=\sigma^{-1} g \sigma \in N$ induit une application birégulière non triviale de l'hyperplan $H_{0}$ dans lui-même, correspondant à $g_{p} \in \operatorname{Aut}\left(\mathbb{P}\left(T_{p}\right)\right)$. D'après le lemme 3.3, il existe dans $N$ un élément $f_{0} \in J_{n}$, qui préserve la fibration rationnelle $\phi: \mathbb{P}_{k}^{n} \rightarrow H_{0}$ donnée par $\left(x_{0}: \cdots: x_{n}\right) \rightarrow\left(0: x_{1}: \cdots: x_{n}\right)$ et agit sur la base du pinceau comme $f_{\left.\right|_{H_{0}}}$, donc de manière non triviale.

Montrons maintenant qu'il existe $\beta \in J_{n}^{0}$ tel que $r=\beta^{-1} f_{0} \beta\left(f_{0}\right)^{-1}$ soit un élément non trivial de $N \cap J_{n}^{0}$. Rappelons que $J_{n}$ est isomorphe au produit semi-direct $J_{n}^{0} \rtimes \operatorname{Bir}\left(k^{n-1}\right)$, et écrivons $f_{0}=(a, b)$ dans ce produit, avec $a \in J_{n}^{0}$ et $b \in \operatorname{Bir}\left(k^{n-1}\right)$ non trivial par construction. Alors, $r$ s'écrit

$$
\left(\beta^{-1}, 1\right) \circ(a, b) \circ(\beta, 1) \circ\left(b^{-1}\left(a^{-1}\right), b^{-1}\right)=\left(\beta^{-1} \cdot a \cdot b(\beta) \cdot a^{-1}, 1\right) .
$$

Par conséquent, $r$ est un élément de $N \cap J_{n}^{0}$, qui est de plus non trivial si et seulement si $\beta \neq a \cdot b(\beta) \cdot a^{-1}$. Si $a$ est l'identité, il suffit de choisir $\beta \in J_{n}^{0}$ non fixé par $b$ (par exemple un élément diagonal donné par une fonction de $k\left(x_{1}, \ldots, x_{n-1}\right)$ qui n'est pas invariante par $\left.b\right)$. Si $a$ n'est pas l'identité, on peut choisir pour $\beta$ un élément de $\operatorname{PGL}(2, k) \subset \operatorname{PGL}\left(2, k\left(x_{1}, \ldots, x_{n-1}\right)\right)$ ne commutant pas avec $a$.

On trouve donc que $N \cap J_{n}^{0}$ est un sous-groupe normal non trivial de $J_{n}^{0} \simeq \operatorname{PGL}\left(2, k\left(x_{1}, \ldots, x_{n-1}\right)\right)$, ce qui implique que $N$ contient $J_{n}^{1} \simeq \operatorname{PSL}\left(2, k\left(x_{1}, \ldots, x_{n-1}\right)\right)$ (voir par exemple [3, Chapitre II, §2]). De plus, comme $J_{n}^{1} \cap \operatorname{Aut}\left(\mathbb{P}_{k}^{n}\right)$ est non trivial et $\operatorname{Aut}\left(\mathbb{P}_{k}^{n}\right) \simeq \operatorname{PGL}(n+1, k)$ est simple, $N$ contient $\operatorname{Aut}\left(\mathbb{P}_{k}^{n}\right)$.

ThÉORÈme 4.2. - Muni de sa topologie, le groupe $\mathrm{Cr}_{2}(k)=\operatorname{Bir}\left(\mathbb{P}_{k}^{2}\right)$ est simple.

Démonstration. - Suit de la proposition précédente et du fait que $\operatorname{Bir}\left(\mathbb{P}_{k}^{2}\right)$ est engendré $\operatorname{par} \operatorname{Aut}\left(\mathbb{P}_{k}^{2}\right)$ et $J_{2}^{1} \simeq \operatorname{PSL}\left(2, k\left(x_{1}\right)\right)$. Démontrons cette dernière partie. On note $\alpha_{1}, \alpha_{2}, \beta_{1}, \beta_{2}$ les éléments de $\mathrm{Cr}_{2}(k)=\operatorname{Bir}\left(\mathbb{P}_{k}^{2}\right)$ suivants (vus ici sur la carte affine $\left(x_{1}, x_{2}\right) \mapsto\left(1: x_{1}: x_{2}\right)$ ):

$$
\begin{array}{ll}
\alpha_{1}:\left(x_{1}, x_{2}\right) \rightarrow\left(x_{1},-\frac{1}{x_{2}}\right) & \beta_{1}:\left(x_{1}, x_{2}\right) \mapsto\left(x_{2}, x_{1}\right) ; \\
\alpha_{2}:\left(x_{1}, x_{2}\right) \rightarrow\left(-\frac{1}{x_{1}}, x_{2}\right) & \beta_{2}:\left(x_{1}, x_{2}\right) \mapsto\left(-x_{1},-x_{2}\right) .
\end{array}
$$

Alors, $\alpha_{1}$ est un élément de $J_{2}^{1}$ et $\beta_{1}, \beta_{2}$ sont deux éléments de $\operatorname{Aut}\left(\mathbb{P}_{k}^{2}\right)$. De plus, $\alpha_{2}=\beta_{1} \alpha_{1} \beta_{1}$ et $\alpha_{1} \alpha_{2} \beta_{2}$ est la transformation quadratique standard. Le résultat se déduit alors du théorème de Noether-Castelnuovo : le groupe $\operatorname{Bir}\left(\mathbb{P}_{k}^{2}\right)$ est engendré par $\operatorname{Aut}\left(\mathbb{P}_{k}^{2}\right)$ et la transformation quadratique standard $\left(x_{1}, x_{2}\right) \rightarrow\left(\frac{1}{x_{1}}, \frac{1}{x_{2}}\right)$ (voir [9, Chapter V, $\S 5$, Theorem 2, p. 100] pour une preuve valable en toute caractéristique).

Remarque 4.3. - La simplicité de $\operatorname{Bir}\left(\mathbb{P}_{k}^{2}\right)$, en tant que groupe abstrait, est toujours ouverte. Pour de plus amples résultats dans cette direction, voir [1] et [4].

\section{Connexité de $\operatorname{Bir}\left(\mathbb{P}_{k}^{n}\right)$}

Comme $\operatorname{Bir}\left(\mathbb{P}_{k}^{2}\right)$ est engendré par $\operatorname{Aut}\left(\mathbb{P}_{k}^{2}\right)$ et $J_{2}^{0}$, le groupe $\operatorname{Bir}\left(\mathbb{P}_{k}^{2}\right)$ est connexe. En dimension supérieure, il n'existe pas d'analogue au théorème de Noether-Castelnuovo (voir [6]) et il ne paraît pas évident a priori de trouver un ensemble adéquat de générateurs. Toutefois, nous pouvons prouver le résultat suivant : 
Théorème 5.1. - Pour tout $n \geq 1$, le groupe $\operatorname{Cr}_{n}(k)=\operatorname{Bir}\left(\mathbb{P}_{k}^{n}\right)$ est linéairement connexe au sens suivant : pour tous $f, g \in \mathrm{Cr}_{n}(k)$, il existe un ouvert $U$ de la droite affine sur $k$ contenant 0 et 1 , et un morphisme $\theta: U \rightarrow \mathrm{Cr}_{n}(k)$ tel que $\theta(0)=f$ et $\theta(1)=g$.

En particulier, le groupe $\mathrm{Cr}_{n}(k)$ est connexe.

Démonstration. - Si $U \subset \mathbb{A}_{k}^{1}$ est un ouvert contenant 0 et 1 et que le morphisme $\theta: U \rightarrow \mathrm{Cr}_{n}(k)$ satisfait $\theta(0)=f$ et $\theta(1)=g$, on dira que $\theta$ joint $f \grave{a} g$; en notant $U^{\prime}$ l'ouvert qui est l'image de $U$ par $t \mapsto 1-t$, le morphisme $U^{\prime} \rightarrow \mathrm{Cr}_{n}(k)$ défini par $t \mapsto \theta(1-t)$ joint $g$ à $f$. De plus, si $\nu: V \rightarrow \mathrm{Cr}_{n}(k)$ joint $g$ à $h$, le morphisme $U \cap V \rightarrow \mathrm{Cr}_{n}(k)$ défini par $t \mapsto \theta(t) \circ g^{-1} \circ \nu(t)$ joint $f$ à $h$. On en déduit que la relation « $f$ et $g$ sont joignables » est une relation d'équivalence.

Notons $\mathcal{U}_{0} \subset \mathrm{Cr}_{n}(k)$ l'ensemble des éléments joignables à l'identité. On observe que $\mathcal{U}_{0}$ est un sous-groupe normal de $\mathrm{Cr}_{n}(k)$. En effet, si $\theta$ joint 1 à $f$, alors $t \mapsto \theta(1-t) \circ f^{-1}$ joint 1 à $f^{-1}$ et si $\nu$ joint 1 à $g$, alors $t \mapsto \theta(t) \circ \nu(t)$ joint 1 à $f \circ g$; de plus, si $h \in \mathrm{Cr}_{n}(k)$, $t \mapsto h \circ \theta(t) \circ h^{-1}$ joint 1 à $h \circ f \circ h^{-1}$.

Montrons maintenant que $\operatorname{Aut}\left(\mathbb{P}_{k}^{n}\right) \simeq \operatorname{PGL}(n+1, k)$ est contenu dans $U_{0}$ (c'est-à-dire qu'il est linéairement connexe). Les éléments de la forme

$$
\left(x_{0}: x_{1}: \cdots: x_{n}\right) \mapsto\left(x_{0}+\sum_{i=1}^{n} a_{0, i} x_{i}: x_{1}+\sum_{i=2}^{n} a_{1, i} x_{i}: \cdots: x_{n-1}+a_{n-1, n} x_{n}: x_{n}\right)
$$

sont joignables à l'identité (remplacer tous les $a_{i, j}$ par $t \cdot a_{i, j}$ donne le morphisme souhaité). De même, un élément diagonal

$$
\left(x_{0}: \cdots: x_{n}\right) \mapsto\left(a_{0} x_{0}: \cdots: a_{n} x_{n}\right)
$$

est joignable à l'identité (remplacer $a_{i} \operatorname{par}\left(a_{i}-1\right) t+1$ donne le morphisme souhaité). Comme ces éléments et leurs conjugués engendrent $\operatorname{Aut}\left(\mathbb{P}_{k}^{n}\right)$, on en déduit que $\operatorname{Aut}\left(\mathbb{P}_{k}^{n}\right) \subset U_{0}$.

Le même argument montre que $J_{n}^{0} \simeq \operatorname{PGL}\left(2, k\left(x_{1}, \ldots, x_{n-1}\right)\right)$ est contenu dans $\mathcal{U}_{0}$.

Pour $n=1, \operatorname{Bir}\left(\mathbb{P}_{k}^{1}\right)=\operatorname{Aut}\left(\mathbb{P}_{k}^{1}\right)$, qui est linéairement connexe. On va alors supposer que $n \geq 2$ et que $\mathrm{Cr}_{n-1}(k)$ est linéairement connexe (en procédant par induction sur $n$ ). Alors le groupe $J_{n}$, engendré par $J_{n}^{0}$ et $\mathrm{Cr}_{n-1}(k)$, est contenu dans $\mathcal{U}_{0}$.

Montrons maintenant que tout élément $g \in \operatorname{Bir}\left(\mathbb{P}_{k}^{n}\right)$ appartient à $U_{0}$, ce qui donnera le résultat souhaité. Quitte à multiplier $g$ par un élément de $\operatorname{Aut}\left(\mathbb{P}_{k}^{n}\right) \subset \mathcal{U}_{0}$, on peut supposer que $g$ ait un point fixe $p$, et que $g$ et son inverse soient régulières en $p$; on supposera de plus après conjugaison que $p=(1: 0: \cdots: 0)$.

Soit $\sigma:\left(x_{0}: \cdots: x_{n}\right) \rightarrow\left(\frac{1}{x_{0}}: \cdots: \frac{1}{x_{n}}\right)$ la transformation standard de $\mathbb{P}_{k}^{n}$ (de degré $n$ ), alors $\sigma$ est une involution qui contracte l'hyperplan $H_{0}$ d'équation $x_{0}=0$ sur le point $p$. L'élément $f=\sigma^{-1} g \sigma$ induit une application birégulière de $H_{0}$ dans lui-même. Le lemme 3.3 nous donne un élément $f_{0} \in J_{n}$ (la dérivée normale de $f$ le long de $H_{0}$ ) tel que $f$ et $f_{0}$ sont joignables; par conséquent $f \in \mathcal{U}_{0}$. Le groupe $\mathcal{U}_{0}$ étant normal dans $\mathrm{Cr}_{n}(k), g$ appartient également à $\mathcal{U}_{0}$. 


\section{RÉFÉRENCES}

[1] V. I. Danilov, Non-simplicity of the group of unimodular automorphisms of an affine plane, Mat. Zametki 15 (1974), 289-293.

[2] M. Demazure, Sous-groupes algébriques de rang maximum du groupe de Cremona, Ann. Sci. École Norm. Sup. 3 (1970), 507-588.

[3] J. A. Dieudonné, La géométrie des groupes classiques, Ergebn. der Math. und ihrer Grenzg. 5, Springer, 1971.

[4] M. H. Gizatullin, The decomposition, inertia and ramification groups in birational geometry, in Algebraic geometry and its applications (Yaroslavl', 1992), Aspects Math. E 25, Vieweg, 1994, 39-45.

[5] D. Mumford, Algebraic geometry, in Mathematical developments arising from Hilbert problems. Proceedings of the Symposium in Pure Mathematics of the American Mathematical Society held at Northern Illinois University, De Kalb, 1974, 44-45.

[6] I. PAN, Une remarque sur la génération du groupe de Cremona, Bol. Soc. Brasil. Mat. (N.S.) 30 (1999), 95-98.

[7] J-P. Serre, Communication personnelle.

[8] J-P. Serre, Le groupe de Cremona et ses sous-groupes finis, Séminaire Bourbaki, vol. 2008/09, exposé n 1000, à paraître dans Astérisque.

[9] I. R. Shafarevich, Algebraic surfaces, Proc. Steklov Inst. Math. 75, 1967.

(Manuscrit reçu le 25 juin 2009;

accepté, après révision, le 19 octobre 2009.)

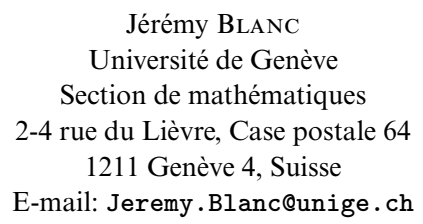

$4^{\mathrm{e}}$ SÉRIE - TOME $43-2010-\mathrm{N}^{\mathrm{o}} 2$ 